\title{
Optimal Nonlinear Estimation for Aircraft Flight Control in Wind Shear*
}

\author{
SANDEEP S. MULGUND $\dagger$ and ROBERT F. STENGEL \\ Nonlinear-inverse-dynamic control combined with optimal state estimation \\ can increase the likelihood that an aircraft will survive an encounter with \\ hazardous microburst wind shear.
}

Key Words-Nonlinear systems; control system design; decoupling; inverse systems; estimation; Kalman filters; aerospace control.

\begin{abstract}
An Extended Kalman Filter (EKF) is developed to estimate the state of a jet transport aircraft. The EKF is based on the nonlinear longitudinal aircraft equations of motion, and it is designed to provide estimates of horizontal and vertical atmospheric wind inputs. The optimal state and disturbance estimates are incorporated in feedback control laws based on the aircraft's nonlinear inverse dynamics. The EKF produces accurate estimates, and the resultant flight trajectories are very similar to those obtained with perfect state feedback. The EKF is sensitive to uncertainty in the dynamic model, but much of the lost performance can bc restored by treating the uncertainty as a random disturbance input.
\end{abstract}

\section{NOMENCLATURE}

$D$ drag;

$E(\cdot)$ expected value;

$E_{\mathrm{s}} \quad$ specific energy;

$F \quad$ nondimensional wind shear hazard index;

$g$ acceleration due to gravity;

$h$ altitude;

$I_{y y} \quad$ moment of inertia about body $y$ axis;

$L \quad$ lift;

$m$ mass;

$M \quad$ pitching moment;

$q$ pitch rate;

$r$ rate of climb;

$R \quad$ radius of downdraft column;

$S \quad$ Laplace variable;

$t \quad$ time;

$T$ engine thrust;

u aircraft control vector;

* Received 10 May 1994; received in final form 3 May 1995. This paper was not presented at any IFAC meeting. This paper was recommended for publication in revised form by Associate Editor Peter Fleming under the direction of Editor Yaman Arkun. Corresponding author Professor Robert F. Stengel. Tel. +1 609258 5103; Fax +1 609258 6109; E-mail stengel@princeton.edu.

† Charles River Analytics Inc., 55 Wheeler St, Cambridge, MA 02138, U.S.A.

$\ddagger$ Department of Mechanical and Aerospace Engineering, Princeton University, Princeton, NJ 08544, U.S.A.
$U_{\max }$ maximum horizontal wind speed;

$V_{\mathrm{a}} \quad$ airspeed;

$w_{x} \quad$ wind component along $x$ axis;

$w_{h} \quad$ wind component along $h$ axis

$W$ weight;

$x \quad$ distance along $x$ axis;

$x \quad$ aircraft state vector;

y control system command vector;

$z_{\max }$ altitude of maximum outflow.

$\alpha \quad$ angle of attack;

$\gamma \quad$ flight path angle;

$\delta_{\mathrm{E}} \quad$ elevator deflection;

$\delta_{\mathrm{T}} \quad$ throttle setting;

$\theta$ pitch attitude.

(c) time derivative;

$\left(^{\wedge}\right.$ estimated quantity.

Subscripts

a, A air-mass-referenced quantity;

c commanded value;

i, I inertially referenced quantity;

\section{INTRODUCTION}

Severe low-altitude wind variability represents an infrequent but significant hazard to aircraft taking off or landing. During the period from 1964 to 1985, microburst wind shear was a contributing factor in at least 26 civil aviation accidents involving nearly 500 fatalities and over 200 injuries (Townsend, 1983). A microburst is a strong localized downdraft that strikes the ground, creating winds that diverge radially from the impact point. The effects of microburst wind shear on airplane dynamics have only recently 
been understood in detail, and it has been found that effective recovery from inadvertent encounteres may require counterintuitive piloting techniques (Hinton, 1988).

The aviation community has initiated an extensive research effort to solve the wind shear problem. The Federal Aviation Administration (FAA) and the National Aeronautics and Space Administration (NASA) have established an integrated program to address the wind shear problem through focused research and development (Bowles, 1989; Hinton, 1989). The FAA's Wind Shear Training Aid (U.S. Department of Transportation, 1987) recommends that on recognizing an encounter with severe wind shear, the pilot should command maximum thrust and rotate the aircraft to an initial target pitch anglc of $15^{\circ}$. This pitch target was identified through rigorous analyses using full six-degree-offreedom flight simulators and wind models representative of actual accident cases (Kupcis, 1990).

Optimal trajectory analysis (OTA) has been used to identify the limits of aircraft performance in wind shear and to determine the control strategies required to achieve such performance (Miele et al., 1990: Mulgund and Stengel, 1993a, b; Psiaki, 1987; Psiaki and Stengel, 1985, 1991: Zhao and Bryson, 1990a). Computation of these trajectories requires global knowledge of the flow field. Since this is not possible in practice. OTA results are not immediately useful for real-time aircraft control. Consequently, feedback control laws employing local wind-field knowledge have been developed for near-optimal flight control (Miele et al., 1990; Zhao and Bryson, 1990b; Miele et al., 1986a, $1986 \mathrm{~b})$.

The goal of this research is to bridge the gap between the performance achieved using OTA and that attainable using feedback control based on local (and possibly forward-look) wind field knowledge. In Mulgund and Stengel (1993c) we presented the design of a feedback control law based on the aircraft's nonlinear inverse' dynamics (NID) (Elgersma and Morton, 1989; Hauser et al., 1989: Lane and Stengel, 1988; Menon et al., 1987, 1991). The NID controller demonstrated safe flight through severe microbursts encountered on the final approach. It was assumed that the aircraft state was known exactly, as were horizontal and vertical wind inputs. The issue of estimating the aircraft state and disturbance inputs from available sensor outputs is the focus of this paper. The extended Kalman filter (EKF) is postulated as a suitable estimator structure, and it is evaluated in concert with the NID control laws.
EFFECT OF WIND SHEAR ON AIRPLANE DYNAMICS

Aircraft model and equations of motion

A three degree-of-freedom model of a twin-jet transport aircraft is used for this study. The aircraft has a gross weight of $85000 \mathrm{lb}$ $(38500 \mathrm{~kg})$ and maximum takeoff thrust of $24000 \mathrm{lb}(107 \mathrm{kN})$. Its aerodynamic coefficients are complex nonlinear functions of altitude, Mach number, incidence angles, rotation rates, control deflections, configuration changes (such as gear and flap deflection), and ground proximity. Effects of wind shear on aircraft motion and aerodynamics are modeled using the techniques described in Frost and Bowles (1984) and Stengel (1990). The relevant reference frames used to describe the aircraft's position, orientation and velocity are presented in Fig. 1. Flight is assumed to take place in a vertical plane over a flat Earth, and a coordinate system fixed to the ground is defined as the inertial reference frame. On the basis of these assumptions, the equations of motion are

$$
\begin{gathered}
\dot{x}=V_{\mathrm{a}} \cos \gamma_{\mathrm{a}}+w_{x}, \\
\dot{h}=V_{\mathrm{a}} \sin \gamma_{\mathrm{a}}+w_{h}, \\
\dot{V}_{\mathrm{a}}=\frac{T}{m} \cos \alpha_{\mathrm{a}}-\frac{D}{m}-g \sin \gamma_{\mathrm{a}} \\
-\dot{w}_{x} \cos \gamma_{\mathrm{a}}-\dot{w}_{h} \sin \gamma_{\mathrm{a}}, \\
\dot{\gamma}_{\mathrm{at}}=\frac{1}{V_{\mathrm{a}}}\left(\frac{T}{m} \sin \alpha_{\mathrm{a}}+\frac{L}{m}-g \cos \gamma_{\mathrm{a}}\right. \\
\left.-\dot{w}_{h} \cos \gamma_{\mathrm{a}}+\dot{w}_{x} \sin \gamma_{\mathrm{a}}\right), \\
\dot{\alpha}_{\mathrm{a}}=q-\dot{\gamma}_{\mathrm{a}}, \\
I_{v y} \dot{q}=M .
\end{gathered}
$$

The effect of wind shear on airplane energy state can be described compactly. First define the specific energy (energy per unit weight) as the sum of air-mass relative kinetic energy and incrtial potential energy:

$$
E_{\mathrm{s}}=\frac{V_{\mathrm{a}}^{2}}{2 g}+h .
$$

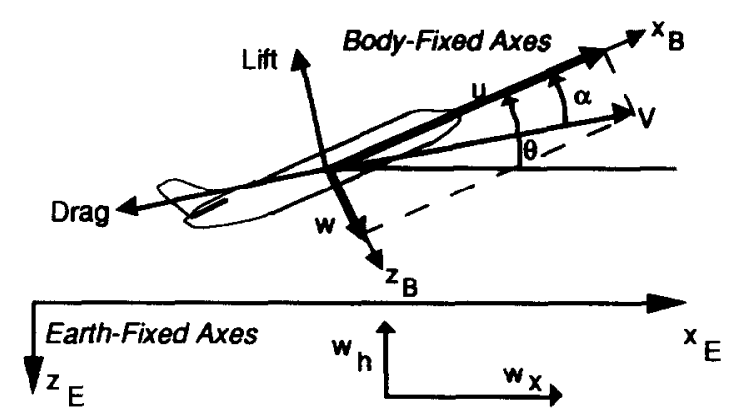

Fig. 1. Coordinate systems and reference frames for flight in wind shear. 
Differentiating this expression and substituting from (1)-(3),

$$
\begin{aligned}
\dot{E}_{\mathrm{s}}= & V_{\mathrm{a}}\left(\frac{T \cos \alpha_{\mathrm{a}}-D}{W}-\frac{\dot{w}_{x}}{g} \cos \gamma_{\mathrm{a}}\right. \\
& \left.-\frac{\dot{w}_{h}}{g} \sin \gamma_{\mathrm{a}}+\frac{w_{h}}{V_{\mathrm{a}}}\right) .
\end{aligned}
$$

The first term is recognizable as the airplane's specific excess power. The three wind terms describe wind shear impact on airplane energy state, and they may be combined into a single scalar quantity called the ' $F$ factor' (Bowles, 1990) as follows:

$$
F=\frac{\dot{w}_{x}}{g} \cos \gamma_{\mathrm{a}}+\frac{\dot{w}_{h}}{g} \sin \gamma_{\mathrm{a}}-\frac{w_{h}}{V_{\mathrm{a}}}
$$

The vertical shear term is typically quite small, and is often neglected. The effect of wind shear on airplane performance is thus expressed as an effective reduction in available specific excess power due to horizontal and vertical shears and downdrafts. Regions where $F$ is negative are considered to be performance-increasing shears, while regions where $F$ is positive are performance-decreasing. $F$ values of more than 0.15 cancel the climb gradient capability of most jet transports.

The aircraft's lift, drag, pitching moment and thrust are expressed as

$$
\begin{gathered}
L=\bar{q} S_{\mathrm{ref}} C_{L} \\
D=\bar{q} S_{\mathrm{ref}} C_{D} \\
T=T_{\max }\left(V_{\mathrm{a}}\right) \delta_{\mathrm{T}}, \quad 0 \leq \delta_{\mathrm{T}} \leq 1, \\
M=\bar{q} S_{\mathrm{ref}} \bar{c} C_{M} .
\end{gathered}
$$

The wind components and spatial gradients used in the equations of motion are obtained from the Oseguera-Bowles downburst model (Oseguera and Bowles, 1988). This analytic time-invariant model represents an axisymmetric stagnation point flow, and it permits simulation of microbursts of varying size and strength through specification of the radius of the downdraft column, the maximum outflow and the altitude of maximum outflow.

\section{NONLINEAR FLIGHT CONTROL}

This description of nonlinear control methods is necessarily brief. More complete treatments can be found in Isidori (1989) and Singh and Rugh (1972). The following derivation is from (Stengel, 1993). Given a system of the form

$$
\dot{\mathbf{x}}=\mathbf{f}(\mathbf{x})+\mathbf{G}(\mathbf{x}) \mathbf{u},
$$

where $\mathbf{x}$ is $n \times 1$ and $\mathbf{u}$ is $m \times 1$, we define an $m$-dimensional output vector

$$
\mathbf{y}=\mathbf{H}(\mathbf{x}) \text {. }
$$

It is possible to construct a nonlinear feedback control law that provides output decoupling of the elements of $\mathbf{y}$ or their derivatives such that $\mathbf{y}^{(d)}=\mathbf{v}$. The new control input $\mathbf{v}$ can be chosen to place the system poles in desired locations (for example, to achieve desired specifications on response overshoot and settling time). The vector $\mathbf{y}^{(d)}$ is expressed as

$$
\mathbf{y}^{(d)}=\mathbf{f}^{*}(\mathbf{x})+\mathbf{G}^{*}(\mathbf{x}) \mathbf{u}=\mathbf{v},
$$

and $d$ is the relative degree of differentiation required to identify a direct control effect on each element of the output vector. The inverse control law then is

$$
\mathbf{u}=\left[\mathbf{G}^{*}(\mathbf{x})\right]^{-1}\left[\mathbf{v}-\mathbf{f}^{*}(\mathbf{x})\right],
$$

and the closed-loop dynamics of the system take the form

$$
\dot{\mathbf{x}}=\mathbf{f}(\mathbf{x})+\mathbf{G}(\mathbf{x})\left[\mathbf{G}^{*}(\mathbf{x})\right]^{-1}\left[\mathbf{v}-\mathbf{f}^{*}(\mathbf{x})\right] .
$$

While the expression of the inverse control law appears simple, its implementation can be quite complex. Evaluation of the functions $\mathbf{f}^{*}(\mathbf{x})$ and $\mathbf{G}^{*}(\mathbf{x})$ requires that a full, $d$-differentiable model of the aircraft dynamics be included in the control system. The controller can be simplified if the system can be partitioned into slow- and fast-time-scale subsystems (Chow and Kokotovic, 1978). The separation of the dynamics into fast and slow time-scales is a natural consequence of the underlying physics. For the aircraft problem, it is assumed that the pitch rate evolves faster than the flight path and velocity. This is consistent with the time-scale separation between the phugoid and short-period modes of an aircraft's longitudinal dynamics (Etkin, 1972). This technique has previously been applied to the flight control problem (Elgersma and Morton, 1989; Hauser et al., 1989; Menon et al., 1987, 1991). In the present study the effects of wind shear are explicitly considered in the inversion.

Three sets of output vectors are considered for approach encounters with wind shear: airspeed/climb rate, groundspeed/climb rate and throttle/climb rate (Mulgund and Stengel, 1993c). A combination of airspeed, groundspeed and climb-rate regulation (Psiaki and Park, 1992) is employed during glide slope tracking on the final approach. When the wind shear $F$ factor (9) exceeds a certain threshold, the controller commands an aborted-landing maneuver using full throttle and a nominal positive climb rate. 
The controller demonstrates good recovery performance in a variety of microburst encounters when perfect state feedback is used as the basis of the control. Such full-state feedback would not be available in practice-it would be necessary to estimate the aircraft statc and disturbance components for noisy sensor outputs. An extended Kalman filter has been developed to accomplish this task, and it is now described.

\section{OPTIMAL STATE ESTIMATION}

An optimal estimator (Gelb, 1974) is at computational algorithm that processes measurements to calculate a minimum-error estimate (in accordance with some stated optimality critcrion) of the state of a dynamic system using:

- knowledge of system and measurement dynanics;

- assumed statistics of system disturbance inputs and measurement errors:

- initial condition information.

Such an algorithm minimizes estimation error in some well-defined statistical manner. and makes use of all of the measurement data and prior knowledge about the system. However, its potential drawback is sensitivity to erroneous system models and statistics. The extended Kalman filter derives optimal aircraft state and wind component estimates for use with the NID control laws. The EKF is an optimal filter in the sense that it minimizes the variance in the estimation error associated with a nonlinear system's linear perturbation model. It is an extension of the widely used Kalman filter (Kalman, 1960), which produces minimumvariance state estimates for linear discrete-timo systems.

The extended Kalman filter computes minimum-variance estimates for nonlinear systems described by the ordinary differential equation

$$
\dot{\mathbf{x}}(t)=\mathbf{f}|\mathbf{x}(t), \mathbf{u}(t), t|+\mathbf{w}(t) .
$$

The vector $\mathbf{f}$ is a nonlinear function of the state $\mathbf{x}$, the deterministic control input $\mathbf{u}$ and time. The disturbance input $\mathbf{w}$ is a white, zero-mean Gaussian random process:

$$
\begin{gathered}
E[\mathbf{w}(t)]=\mathbf{0}, \\
E\left[\mathbf{w}(t) \mathbf{w}^{\mathrm{T}}(\tau)\right]=\mathbf{Q}_{\mathrm{c}}(t) \delta(t-\tau),
\end{gathered}
$$

where $E($.$) denotes the expected value of the$ function. The disturbance is thus characterized by its spectral density matrix $\mathbf{Q}_{c}(t)$. The quantity $\delta$ is the Dirac delta function:

$$
\begin{gathered}
\delta(t-\tau)= \begin{cases}\infty & (t=\tau), \\
0 & (t \neq \tau),\end{cases} \\
\lim _{t \rightarrow \infty} \int_{t}^{t+t} \delta(t-\tau) \mathrm{d} \tau=1 .
\end{gathered}
$$

Both continuous-time and discrete-time measurements can be handled; considered here is the case of discrete measurements of the form

$$
\mathbf{z}\left(t_{k}\right)=\mathbf{h}\left[\mathbf{x}\left(t_{k}\right)\right]+\mathbf{n}_{k}, \quad k=0,1,2, \ldots .
$$

The measurement noise $\mathbf{n}$ also is assumcd to be a white, zero-mean Gaussian random process that is uncorrelated with the disturbance input:

$$
\begin{gathered}
E\left(\mathbf{n}_{k}\right)=\mathbf{0}, \\
E\left(\mathbf{n}_{k} \mathbf{n}_{k}^{\mathrm{T}}\right)=\mathbf{R}_{k}, \\
E\left[\mathbf{w}(t) \mathbf{n}_{k}^{\mathrm{T}}\right]=\mathbf{0} \quad \text { for all } k \text { and all } t .
\end{gathered}
$$

The expected values of the initial state and its covariance are assumed known:

$$
\begin{gathered}
E\left(\mathbf{x}_{0}\right)=\hat{\mathbf{x}}_{(j,} \\
E\left[\left(\mathbf{x}_{0}-\hat{\mathbf{x}}_{0}\right)\left(\mathbf{x}_{0}-\hat{\mathbf{x}}_{0}\right)^{\mathrm{T}}\right]=\mathbf{P}_{0} .
\end{gathered}
$$

Before presenting the equations for the computation of the state estimate and its covariance. it is necessary to define the sensitivity matrices:

$$
\begin{gathered}
\mathbf{F}(t)=\left.\mathbf{F}[\mathbf{x}(t), \mathbf{u}(t), t] \equiv \frac{\partial \mathbf{f}[\cdot]}{\partial \mathbf{x}}\right|_{\mathbf{x}(t)=\hat{\mathbf{x}}(t)}, \\
\mathbf{H}(t)=\left.\mathbf{H}[\mathbf{x}(t)] \equiv \frac{\partial \mathbf{h}[\cdot]}{\partial \mathbf{x}}\right|_{\mathbf{x}(t)=\hat{\mathbf{x}}(t)} .
\end{gathered}
$$

The EKF propagates (or extrapolates) both the state estimate and its covariance using the system model. New measurements are combined with the extrapolated estimate to generate an 'updated' estimate. The state covariance also is updated to reflect the information contained in the measurement. A weighting factor called the Kalman filter gain is used to combine the extrapolated estimate with the new measurement. This gain is defined in such a way that it minimizes the estimation error covariance after the update. It is useful to introduce notation that distinguishes state estimates made before and after the measurement updates: $\hat{\mathbf{x}}_{k}(-)$ is the state estimate resulting from extrapolation alone (i.e. before the observation $\mathbf{z}_{k}$ is considered), and $\hat{\mathbf{x}}_{k}(+)$ is the corrected estimate that 
accounts for the measurements. The pre- and post-measurement covariance matrices $\mathbf{P}_{k}(-)$ and $\mathbf{P}_{k}(+)$ are defined similarly. The state and covariance estimates are extrapolated using

$$
\hat{\mathbf{x}}\left[t_{k}(-)\right]=\hat{\mathbf{x}}\left[t_{k-1}(+)\right]+\int_{t_{k-1}}^{t_{k}} \mathbf{f}\{\hat{\mathbf{x}}[\tau(-)], \mathbf{u}(\tau), \tau\} \mathrm{d} \tau,
$$

$$
\begin{aligned}
& \mathbf{P}\left[t_{k}(-)\right]=\mathbf{P}\left[t_{k-1}(+)\right] \\
& \quad+\int_{\tau_{k-1}}^{t_{k}}\left[\mathbf{F}(\tau) \mathbf{P}(\tau)+\mathbf{P}(\tau) \mathbf{F}(\tau)^{\mathrm{T}}+\mathbf{Q}_{c}(\tau)\right] \mathrm{d} \tau .
\end{aligned}
$$

The Jacobian $\mathbf{F}(\tau)$ is evaluated in the interval for corresponding values of $\hat{\mathbf{x}}[\tau(-)]$. The state and covariance are updated as

$$
\hat{\mathbf{x}}_{k}\left[t_{k}(+)\right]=\hat{\mathbf{x}}_{k}\left[t_{k}(-)\right]+\mathbf{K}\left(t_{k}\right)\left\{\mathbf{z}\left(t_{k}\right)-\mathbf{h}\left[\hat{\mathbf{x}}\left[t_{k}(-)\right]\right]\right\},
$$

$$
\mathbf{P}_{k}\left[t_{k}(+)\right]=\left\{\mathbf{I}_{n}-\mathbf{K}\left(t_{k}\right) \mathbf{H}\left(t_{k}\right)\right\} \mathbf{P}\left[t_{k}(-)\right] \text {. }
$$

The filter gain $\mathbf{K}\left(t_{k}\right)$ is

$$
\begin{aligned}
\mathbf{K}\left(t_{k}\right)= & \mathbf{P}\left[t_{k}(-)\right] \mathbf{H}^{\mathrm{T}}\left(t_{k}\right) \\
& \times\left\{\mathbf{H}\left(t_{k}\right) \mathbf{P}\left[t_{k}(-)\right] \mathbf{H}^{\mathrm{T}}\left(t_{k}\right)+\mathbf{R}\left(t_{k}\right)\right\}^{-1} .
\end{aligned}
$$

The matrix $\mathbf{H}\left(t_{k}\right)$ is evaluated using $\mathbf{x}=\hat{\mathbf{x}}\left[t_{k}(-)\right]$. Equations (30)-(36) constitute the EKF algorithm for continuous-time nonlinear systems with discrete measurements.

\section{Filter equations for the jet transport}

The EKF estimates the state of the jet transport aircraft using available inertial and air-data measurements. A key feature of the EKF in this application is its ability to estimate the horizontal and vertical wind components $w_{x}$ and $w_{h}$, along with their first and second time derivatives. Estimates of these quantities are used as the basis of feedback control using the NID control laws. Before presenting the EKF equations for the jet transport, it is worthwhile to briefly examine conventional methods of in-flight wind disturbance estimation.

The Federal Aviation Administration stipulated that all commercial aircraft be outfitted with reactive wind shear detection systems by December, 1993. Typically, these systems compute an estimate of the $F$ factor by differencing inertial and air-relative acceleration signals (Hansen, 1987; Johnson, 1986), since any difference between air-relative and inertial acceleration must be due to unsteady motion of the airmass itself. An algorithm developed by Oseguera et al. (1992) is based on a threedimensional expression of the $F$ factor, and it provides good estimates of $F$ in many different flight conditions. This algorithm has become a measurement standard for the evaluation of forward-looking wind shear detectors. However, this method is not suitable for use with the NID control laws developed in this paper, since it does not derive $w_{x}, w_{h}$ and their first two time derivatives from the $F$ estimate.

Bossi and Bryson (1981) examined the use of constant-gain Kalman Filters for disturbance estimation and detection of engine failure for a short-takeoff and landing (STOL) aircraft during the landing mancuver. The estimator is based upon a linearized model of open-loop aircraft dynamics, and their results suggest that it is possible to develop accurate estimates of horizontal and vertical winds (along with their first time derivatives) by defining $w_{x}, w_{h}, \dot{w}_{x}$ and $\dot{w}_{h}$ as state elements and including them in the filter. This is the approach taken in the design of an EKF for the jet transport.

The wind-axis equations of motion (1)-(6) are used to define the plant model for the estimator equations. There is a first-order lag in powerplant dynamics:

$$
\dot{T}=\frac{T_{\mathrm{c}}-T}{\tau},
$$

where $T_{\mathrm{c}}$ is the commanded thrust, $T$ is the actual thrust and $\tau=2 \mathrm{~s}$. The aircraft state vector is defined nominally as

$$
\mathbf{x}_{\mathrm{ac}}^{\mathrm{T}}=\left[\begin{array}{lllllll}
x & h & V_{\mathrm{a}} & \gamma_{\mathrm{a}} & \alpha_{\mathrm{a}} & q & T
\end{array}\right] .
$$

The control input to the system is

$$
\mathbf{u}=\left[\begin{array}{c}
T_{\mathrm{c}} \\
\delta_{\mathrm{E}}
\end{array}\right] .
$$

The NID control laws also require feedback of $w_{x}, w_{h}, \dot{w}_{x}, \dot{w}_{h}, \ddot{w}_{x}$ and $\ddot{w}_{h}$. It is necessary to make these six variables part of the system state vector. The 'wind state' vector is defined as

$$
\mathbf{x}_{\mathrm{d}}^{\mathrm{T}}=\left[\begin{array}{llllll}
w_{x} & w_{h} & \dot{w}_{x} & \dot{w}_{h} & \ddot{w}_{x} & \ddot{w}_{h}
\end{array}\right]
$$

In order to construct the system model, it is necessary to define the 'dynamics' associated with the wind inputs. Any mathematical model of the wind dynamics represent an approximation to the conditions that exist within a microburst. Error in the modeling can lead to biases in the estimates and even divergence from the actual values. The simplest approach is to use an integral-state model (Bossi and Bryson, 1981) to represent the wind components and their time derivatives. The equation describing the wind dynamics is

$$
\dot{\mathbf{x}}_{\mathrm{d}}=\mathbf{F}_{\mathrm{d}} \mathbf{x}_{\mathrm{d}}+\mathbf{w},
$$


where

$$
\mathbf{F}_{d}=\left[\begin{array}{cccccc}
0 & 0 & 1 & 0 & 0 & 0 \\
0 & 0 & 0 & 1 & 0 & 0 \\
0 & 0 & 0 & 0 & 1 & 0 \\
0 & 0 & 0 & 0 & 0 & 1 \\
0 & 0 & 0 & 0 & 0 & 0 \\
0 & 0 & 0 & 0 & 0 & 0
\end{array}\right], \quad \mathbf{w}=\left[\begin{array}{c}
0 \\
0 \\
0 \\
0 \\
w_{1} \\
w_{2}
\end{array}\right],
$$

and $\mathbf{w}$ is (by assumption) a white, zero-mean Gaussian random variable:

$$
\begin{gathered}
E[\mathbf{w}(t)]=\mathbf{0}, \\
E\left[\mathbf{w}(t) \mathbf{w}^{\mathrm{T}}(\tau)\right]=\mathbf{Q}_{\mathrm{c}}(t) \delta(t-\tau) .
\end{gathered}
$$

The aircraft dynamics may thus be expressed as

$$
\dot{\mathbf{x}}_{\mathrm{ac}}=\mathbf{f}\left(\mathbf{x}_{\mathrm{ac}}, \mathbf{u}, \mathbf{x}_{\mathrm{d}}\right),
$$

where the elements of $\mathbf{f}(\cdot)$ are constructed using (1)-(6). The complete state vector for the estimator plant model is defined as

$$
\chi \triangleq\left[\begin{array}{l}
\mathbf{x}_{\mathrm{ac}} \\
\mathbf{x}_{\mathrm{d}}
\end{array}\right] \text {. }
$$

The combined aircraft/disturbance dynamics are then written as

$$
\dot{\chi}=\left[\begin{array}{c}
\dot{\mathbf{x}}_{\mathrm{ac}} \\
\dot{\mathbf{x}}_{\mathrm{d}}
\end{array}\right]=\left[\begin{array}{c}
\mathbf{f}\left(\mathbf{x}_{\mathrm{ac}}, \mathbf{u}, \mathbf{x}_{\mathrm{d}}\right) \\
\mathbf{F}_{\mathrm{d}} \mathbf{x}_{\mathrm{d}}
\end{array}\right]+\left[\begin{array}{c}
\mathbf{0} \\
\mathbf{w}
\end{array}\right] .
$$

Equation (47) describes the assumed system model used to propagate the EKF. The disturbance input to this system is the vector $w$, whose nonzero components physically represent the third time-derivatives of $w_{x}$ and $w_{h}$. Thus, for the purposes of the EKF, $\dddot{w}_{x}$ and $\dddot{w}_{h}$ are modeled as zero-mean Gaussian random variables. There is a question of how to choose the elements of the matrix $\mathbf{Q}_{c}$, (44). Empirical data on microbursts generally include information on wind speeds, spatial extent and (possibly) $F$ factor; it is difficult to infer anything substantive about the third time derivative of the wind speed components. Consequently, it has been necessary to rely on trial-and-error methods to identify suitable clements of $\mathbf{Q}_{c}$. In effect, its components become design parameters that are adjusted to tune the filter's response. The numerical values used are presented along with the simulation results.

The measurement vector $\mathbf{z}$ consists of the sensor outputs that are available on a typical modern jet transport aircraft. These are altitude (ft), groundspeed ( $\mathrm{ft} \mathrm{s}^{-1}$ ), airspeed ( $\mathrm{ft} \mathrm{s}^{-1}$ ), angle of attack (deg), pitch angle (deg) and rate $\left(\operatorname{deg} \mathrm{s}^{-1}\right)$, altitude rate (ft $\left.\mathrm{s}^{-1}\right)$, and horizontal and vertical acceleration $\left(\mathrm{ft} \mathrm{s}^{-2}\right)$. The vector $\mathbf{z}$ is thus defined as

$$
\mathbf{z}^{\mathrm{T}}=\left[\begin{array}{lllllllll}
h & V_{\mathrm{i}} & V_{\text {a }} & \alpha_{\mathrm{i}} & \boldsymbol{\theta} & q & \dot{h} & \ddot{x} & \ddot{h}
\end{array}\right] .
$$

The measurement noise covariance matrix is defined as

$$
\begin{array}{r}
\mathbf{R}=\operatorname{diag}\left(5^{2}, 3.6^{2}, 1.7^{2}, 0.5^{2}, 0.05^{2},\right. \\
\left.0.05^{2}, 0.5^{2}, 0.322^{2}, 0.322^{2}\right) .
\end{array}
$$

These values are representative of state-of-theart inertial and air-data systems.

The EKF is now evaluated in conjunction with the NID control laws. The EKF/NID performance is compared with that achieved using perfect state feedback. This permits an assessment of how much performance is 'lost' when optimal state estimates based on noisy measurements are used as the basis for the feedback.

\section{SIMULATION RESULTS}

Aircraft encounters with microburst wind shear are considered on the final approach, during which a decision is made to abort the landing and execute a climb-out. A climb rate scheduling strategy (Hinton, 1988) is used to compute the target climb rate during the aborted-landing maneuver as a function of available performance. This produces trajectories that exchange altitude for airspeed in a manner dependent on microburst strength, similar to previously obtained trajectory optimization results (Mulgund and Stengel, 1993b).

The disturbance input spectral density matrix $\mathbf{Q}$. is set to

$$
\mathbf{Q}_{\mathrm{c}}=\operatorname{diag}(0,0,0,0,0.01,0.01) .
$$

The zero off-diagonal components imply that the horizontal and vertical wind inputs are (by assumption) uncorrelated with one another. The nonzero diagonal elements were determined through trial-and-error. If they were made too large, the EKF did a poor job of attenuating measurement noise in its derivation of state and disturbance estimates. Very small diagonal elements resulted in good measurement noise attenuation, but introduced significant lags into the estimates of many of the state components. This estimator lag led to degraded controller performance. Numerical instabilities also were encountered in the propagation of the state and covariance estimates when small numbers were used as the diagonal elements of $\mathbf{Q}_{c}$. The selected numbers provide a good balance between attenuating measurement noise and minimizing estimator lag.

The simulation results are organized to facilitate a comparison of the EKF/NID flight trajectory with that obtained using perfect state feedback, and to illustrate the estimation performance of the EKF. State estimation errors are shown together with the ' $2 \sigma$ ' error estimate, which is computed from the covariance matrix 
$\mathbf{P}(t)$. The $\pm 2 \hat{\sigma}_{i}(t)$ curves provide the estimated $95 \%$ confidence interval on the error associated with the state estimate $\hat{\chi}_{i}(t)$. The aircraft is initialized on the glide slope at a point well outside the microburst core with an initial groundspeed of $245 \mathrm{ft} \mathrm{s}^{-1}\left(75 \mathrm{~m} \mathrm{~s}^{-1}\right)$. The microburst has a core radius of $3000 \mathrm{ft}(914 \mathrm{~m})$ a maximum horizontal wind speed of $70 \mathrm{ft} \mathrm{s}^{-1}$ $\left(21 \mathrm{~m} \mathrm{~s}^{-1}\right)$ and a maximum outflow altitude of $150 \mathrm{ft}(46 \mathrm{~m})$. The controller initiates the aborted-landing maneuver once the estimate of the $F$ factor exceeds a threshold of 0.075 . The initial state estimate is set equal to the actual state, so that $\chi\left(t_{0}\right)=\chi\left(t_{0}\right)$. The covariance matrix is initialized as an identity matrix of appropriate dimension.

Figures 2 and 3 present airspeed and angle of attack versus range from microburst core in both the NID-only and the EKF/NID trajectories. Figure 4 shows the horizontal and vertical wind components experienced by the aircraft in the EKF/NID trajectory.

It is evident from Figs 2 and 3 that the $\mathrm{EKF} / \mathrm{NID}$ trajectory is qualitatively similar to the onc obtained using perfect state fecdback. The aborted-landing maneuver entails the use of low attack angles early in the trajectory, followed by a gradual increase that ceases near the end of the microburst core region. The EKF's ability to estimate the wind components is illustrated in Figs 5 and 6, which present the $w_{x}$ and $w_{h}$ estimation errors, along with the $2 \sigma$ error bounds. The actual and estimated $F$ factors in the EKF/NID trajectory are shown together in Fig. 7. The peak $F$ experienced by the aircraft is approximately 0.4 , indicating that this is a very severe microburst.

It is apparent that the EKF estimates the wind components accurately. The predicted $2 \sigma$ error bounds are good indicators of the EKF's accuracy, implying that the actual filter performance is consistent with the expected performance. The $F$-factor estimate derived from the optimal state and disturbance estimates also is quite accurate.

The results show that NID control laws can

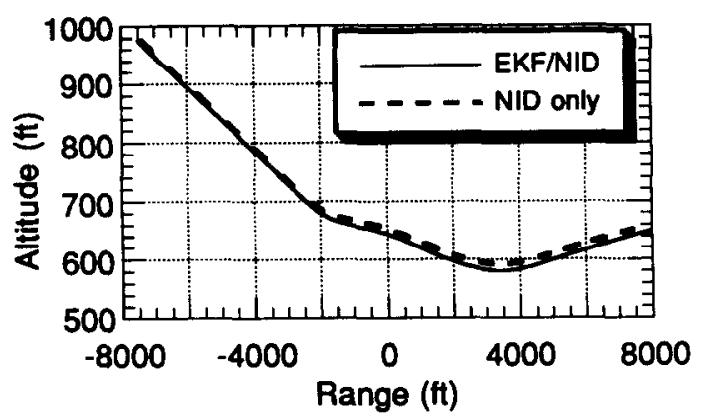

Fig. 2. Altitude versus range during a microburst encounter.

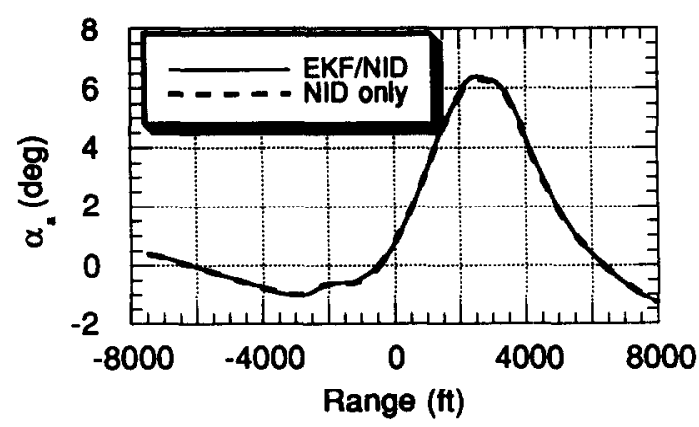

Fig. 3. Angle of attack versus range during a microburst encounter.

perform quite well using optimal state esimates derived from a realistic set of measurements. However, it has been assumed that the initial conditions are known exactly and that there are no modeling errors in the representation of the aircraft aerodynamics used by the EKF/NID pair. Initial condition errors result in a negligible reduction in controller-estimator performance; however, uncertainty in the aerodynamic model poses a serious problem. It has been assumed thus far that the aerodynamic model is exact, and that any inaccuracies in calculating lift, drag and pitching moment are due only to the errors in the state estimates themselves.

The effect of uncertainty in the aerodynamic model is examined by intentionally adding an error to the lift and drag estimates used by the EKF/NID pair. The simulation is repeated using the same microburst parameters as in Figs 2-7, but now a $10 \%$ error is added to the lift and drag estimates used by both the EKF and the NID control laws. Figures 8 and 9 present altitude and angle of attack versus range in the resultant trajectory. For comparison purposes, the response obtained using the NID control law with perfect state feedback and an exact aerodynamic model is also shown. Wind component estimation errors in the EKF/NID trajectory are shown in Figs 10 and 11.

It is apparent that the modeling errors produce a dramatic departure from the nominal flight path (as defined by the NID-only

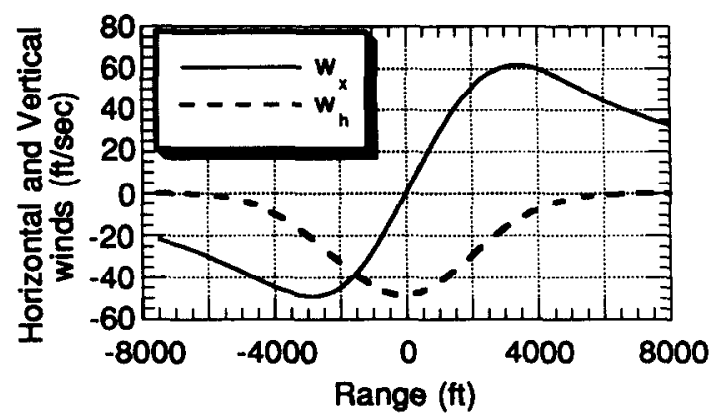

Fig. 4. $w_{x}$ and $w_{h}$ versus range during a microburst encounter, with EKF/NID control. 


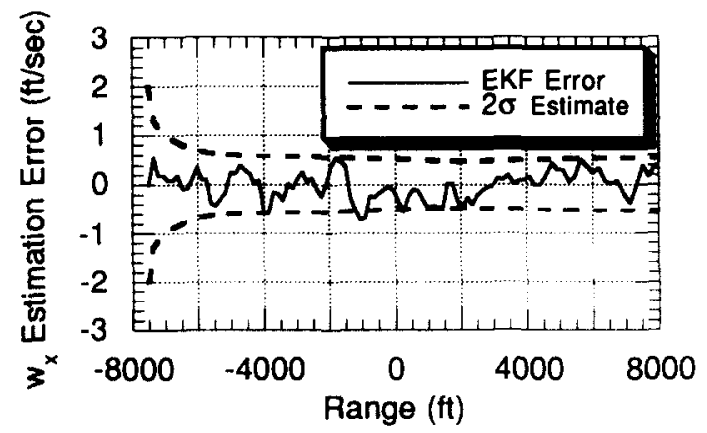

Fig. 5. $w_{x}$ estimation error during a microburst encounter with EKF/NID control.

trajectory). The minimum altitude in the EKF/NID trajectory is some $175 \mathrm{ft}(53 \mathrm{~m})$ lower than in the NID-only trajectory. The aircraft does not track the desired approach path properly in the descent portion of the trajectory. Figures 10 and 11 show that the predicted $2 \sigma$ error bounds are not good indicators of the EKF's performance. The $w_{x}$ estimation error appears to grow without bound.

Uncertainty in the aerodynamic model appears to be a key difficulty in the implementation of the EKF/NID estimator-controller pair. Since it is unlikely that an aircraft's aerodynamic model will ever be known exactly, it is necessary to devise a means of accommodating this uncertainty into the design. One such method is now discussed.

\section{Effect of adding fictitious process noise}

A simple solution for accommodating uncertainty into the design of an EKF is to add fictitious process noise $\mathbf{w}_{\mathrm{f}}(t)$ (Gelb, 1974) to the system model. In effect, uncertainty in the model is treated as a Gaussian random input. The aircraft state equations used by the EKF are modified by adding fictitious disturbance inputs to those terms that depend on lift $L$ or drag $D$. The resultant model is expressed as

$$
\dot{\chi}=\left[\begin{array}{c}
\dot{\mathbf{x}}_{\mathrm{ac}} \\
\dot{\mathbf{x}}_{\mathrm{d}}
\end{array}\right]=\left[\begin{array}{c}
\mathbf{f}\left(\mathbf{x}_{\mathrm{ac}}, \mathbf{u}, \mathbf{x}_{\mathrm{d}}\right) \\
\mathbf{F}_{\mathrm{d}} \mathbf{x}_{\mathrm{d}}
\end{array}\right]+\left[\begin{array}{c}
\mathbf{w}_{\mathrm{f}} \\
\mathbf{w}
\end{array}\right]
$$

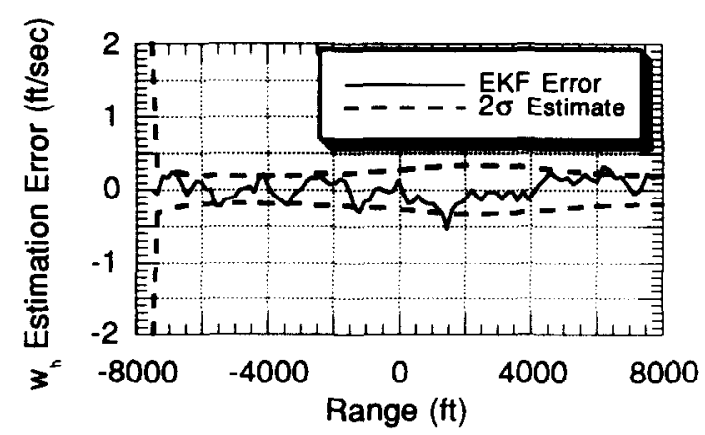

Fig. 6. $w_{h}$ estimation error during a microburst encounter. with EKF/NID control.

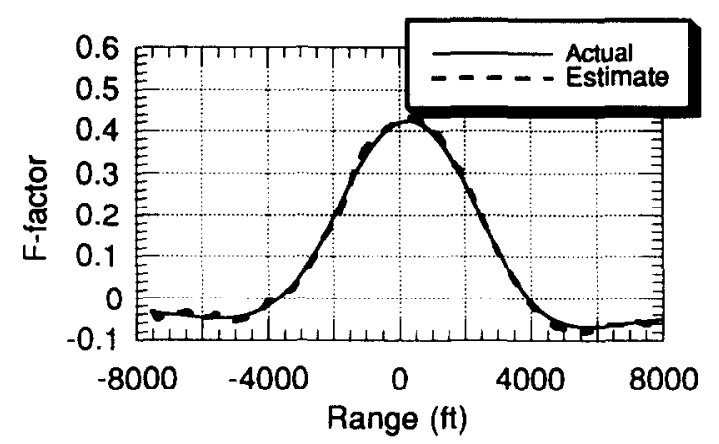

Fig. 7. Actual and estimated $F$ factor during a microburst encounter, with EKF/NID control.

where the fictitious input $\mathbf{w}_{f}(t)$ is a Gaussian, zero-mean random variable:

$$
\begin{gathered}
E\left[\mathbf{w}_{\mathrm{f}}(t)\right]=\mathbf{0}, \\
E\left[\mathbf{w}_{\mathrm{f}}(t) \mathbf{w}_{\mathrm{f}}^{\mathrm{T}}(\tau)\right]=\mathbf{Q}_{\mathrm{f}}(t) \delta(t-\tau) .
\end{gathered}
$$

The elements of $\mathbf{Q}_{\mathrm{f}}$ are chosen to reflect the uncertainty in the time rates of change of the components of $\mathbf{x}_{\mathrm{ac}}$ that depend on $L$ or $D$. For the simulation results that follow, $\mathbf{Q}_{\mathrm{f}}$ is set to

$$
\begin{array}{r}
\mathbf{Q}_{\mathrm{f}}=\operatorname{diag}(0,0,0.2,0.05,0.05, \\
0.2,0.05,0.05,0,0) .
\end{array}
$$

These values were chosen by estimating the effect of an instantaneous $10 \%$ increase in lift and drag on $\dot{\mathbf{x}}_{\mathrm{ac}}$ in a typical steady flight condition. The diagonal elements of $\mathbf{Q}_{\mathrm{f}}$ were first set equal to the magnitude of the change in $\dot{\mathbf{x}}_{\text {aci }}$, and then refined to achieve satisfactory EKF/NID performance.

The EKF/NID trajectory is now recomputed using fictitious process noise in the EKF plant model. As in the previous simulation, $10 \%$ errors are added to the lift and drag estimates used by the EKF/NID pair. Figures 12 and 13 show the resultant altitude and angle of attack response respectively. Once again, the profiles obtained using the NID control law with perfect state feedback and an exact model are shown

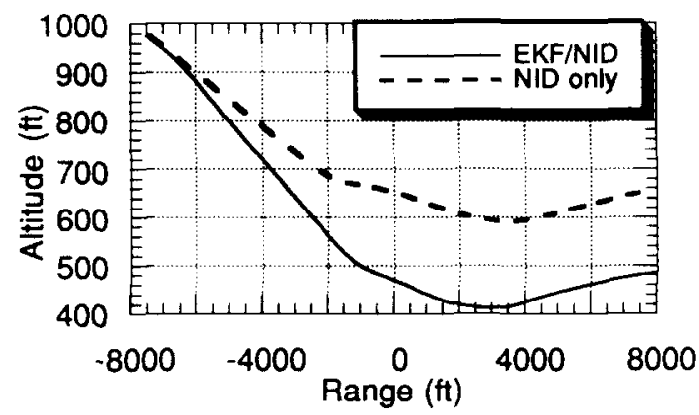

Fig. 8. Altitude versus range during a micorburst encounter, with EKF/NID control and $10 \%$ error in lift and drag estimates. 


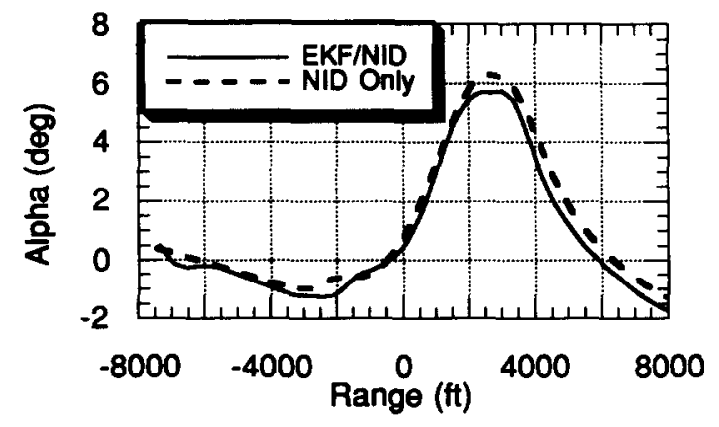

Fig. 9. Angle of attack versus range during a microburst encounter, with EKF/NID control and $10 \%$ error in lift and drag estimates.

for comparison purposes. Horizontal and vertical wind estimation errors in the EKF/NID flight path are shown in Figs 14 and 15.

Figures 12 and 13 show that the EKF/NID altitude and angle of attack profiles with fictitious process noise are much more similar to the NID baseline than those in Figs 8 and 9 . Figures 14 and 15 demonstrate that adding the fictitious process noise restores the EKF's performance. The predicted $2 \sigma$ error bounds are once again good indicators of the accuracy of the wind component estimates. The steady-state error bounds are larger in Figs 14 and 15 than in Figs 10 and 11 . This may be expected, since the

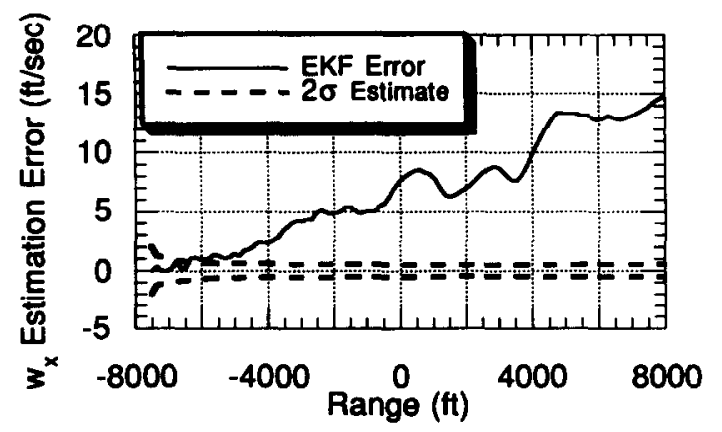

Fig. 10. $w_{x}$ estimation error in the EKF/NID trajectory during a micorburst encounter, with EKF/NID control and $10 \%$ error in lift and drag estimates.

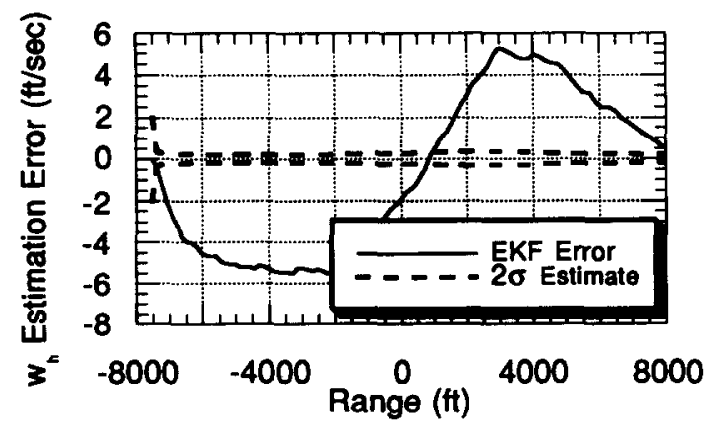

Fig. 11. $w_{h}$ estimation error in the EKF/NID trajectory during a microburst encounter, with EKF/NID control and $10 \%$ error in lift and drag estimates.

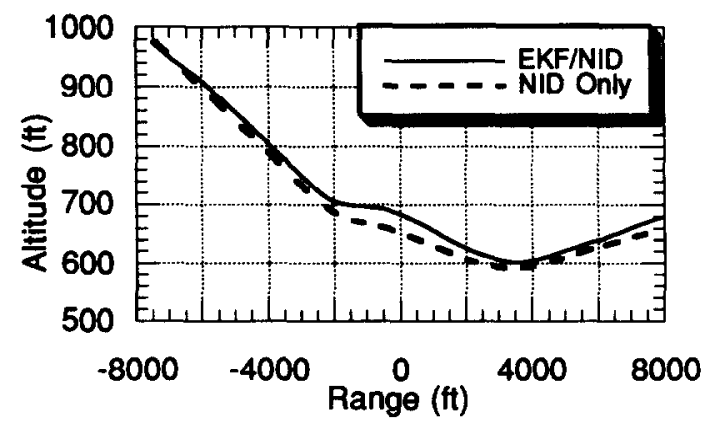

Fig. 12. Altitude versus range during a microburst encounter, with EKF/NID control that incorporates fictitious process noise to account for $10 \%$ error in lift and drag estimates.

EKF plant model now contains uncertainty in the model itself as well as in the measurements.

The simulation results indicate that it is possible to effectively compensate for plant model uncertainty by adding fictitious process noise to the EKF equations. The resultant flight path is similar (but not identical) to the one obtained using perfect state feedback with an exact aerodynamic model. The EKF/NID pair function effectively using a realistic set of measurements and an uncertain aerodynamic model.

Although the computational burden associated with nonlinear inverse-dynamic control is greater

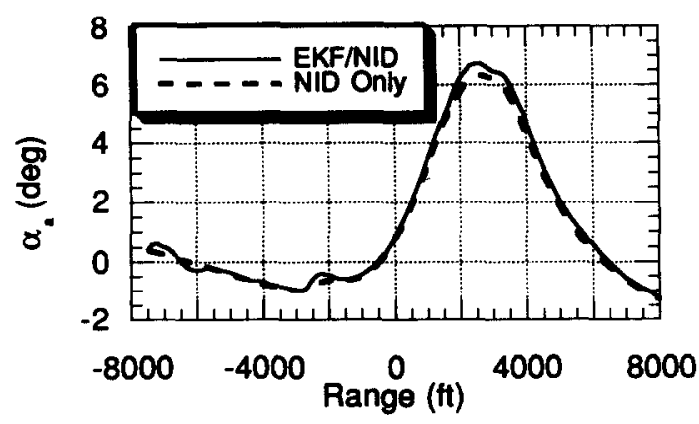

Fig. 13. Angle of attack versus range during a microburst encounter, with EKF/NID control that incorporates fictitious process noise to account for $10 \%$ error in lift and drag estimates.

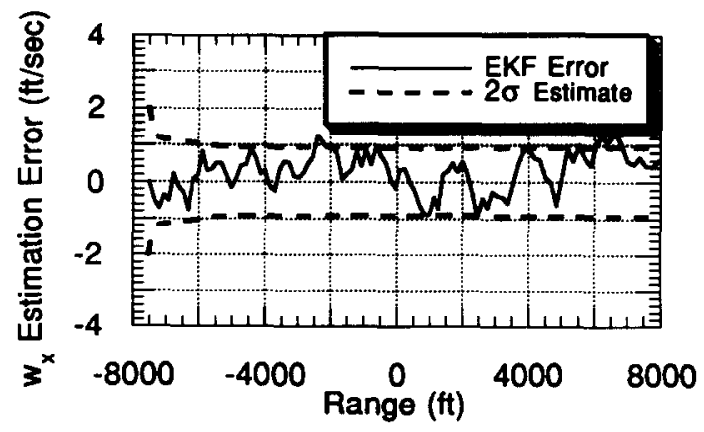

Fig. 14. $w_{x}$ estimation error during a microburst encounter, with EKF/NID control that incorporates fictitious process noise to account for $10 \%$ error in lift and drag estimates. 
than that of constant-gain feedback control, we do not anticipate problems with real-time implementation. When implemented in parallel on three 80286 processors, the NID controller presented in Lane and Stengel (1988) demonstrated real-time performance. As EKF and NID computational requirements are roughly comparable, and current processors are at least an order of magnitude faster than processors of the mid-1980s, future flight-control processors should easily execute the EKF/NID logic.

\section{CONCLUSIONS}

An extended Kalman filter has been developed to estimate the state vector and wind disturbance inputs of a jet transport aircraft. The EKF was evaluated in concert with nonlinear control laws developed previously. Simulated flight trajectories produced using the EKF/NID pair were almost identical to those obtained using the NID control laws with perfect state feedback. The EKF produced accurate estimates of both horizontal and vertical wind inputs using a simple integral-state-model representation of the wind 'system'. This representation makes no assumptions about the structure of the atmospheric disturbance, and it should be able to provide accurate disturbance estimates in a variety of atmospheric conditions.

A key difficulty for applying nonlinear control and estimation techniques is uncertainty in the plant model. The use of an erroneous aerodynamic model in the EKF/NID pair led to inaccurate state estimates and poor command tracking. The introduction of fictitious process noise in the EKF equations, which treated the uncertainty as a random disturbance input, restored most of the lost performance. This result is encouraging from a practical standpoint, because an exact aerodynamic model is not always available for control system design.

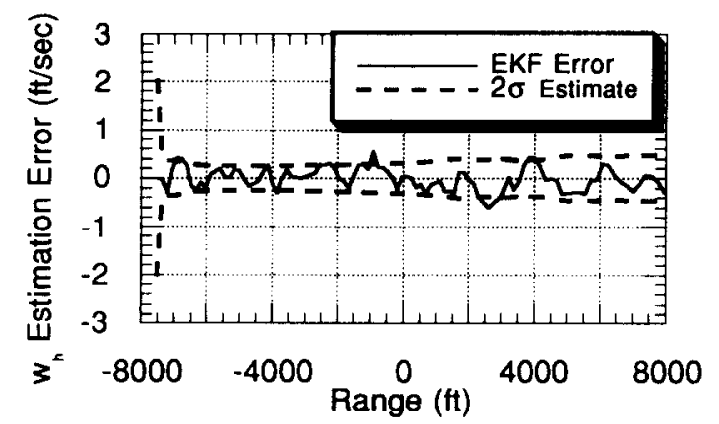

Fig. 15. $w_{h}$ estimation error during a microburst encounter with EKF/NID control that incorporates fictitious process noise to account for $10 \%$ error in lift and drag estimates.
Consequently, the EKF/NID control law is a good candidate for operational implementation.

\section{REFERENCES}

Bossi, J. and A. E. Bryson (1981). Disturbance estimation for a STOL transport during landing. AIAA Paper 81-0018, Presented at the 19th Aerospace Sciences Meeting, St Louis, MO.

Bowles, R. L. (1990). Reducing windshear risk through airborne systems technology. Presented at the 17th Congress of the International Council of the Aeronautical Sciences, Stockholm, 1990.

Chow, J. and P. Kokotovic (1978). Two-time-scale feedback design of a class of nonlinear systems. IEEE Trans. Autom. Control, AC-23, No. 3 .

Elgersma, M. and B. Morton (1989). Partial inversion of noninvertible nonlinear aircraft models. Honeywell Systems Research Center, Minneapolis.

Etkin, B. (1972). Dynamics of Atmospheric Flight. Wiley, New York

Frost. W. and R. Bowles (1984). Wind shear terms in the equations of aircraft motion. J. Aircraft, 21, 866-872.

Gelb, A. (Ed.) (1974). Applied Optimal Estimation. MIT Press, Cambridge, MA.

Hansen, R. (1987). Boeing windshear systems. AIAA Paper $87-2342$.

Hauser, J., S. Sastry and G. Meyer (1989). Nonlinear control design for slightly non-minimum phase systems: application to V/STOL aircraft. Presented at IFAC Conf. NOLCOS '89, Capri.

Hinton, D. A. (1988). Flight management strategies for escape from microburst encounters. NASA TM 4057. Washington, DC.

Hinton, D. A. (1989). Piloted-simulation evaluation of recovery guidance for microburst wind shear encounters. NASA TP 2886, Washington, DC.

Isidori, A. (1989). Nonlinear Control Systems. SpringerVerlag, Berlin.

Johnson, B. (1986). Sensing windshear. Sensors.

Kalman, R. E. (1960). A new approach to linear filtering and prediction problems. Trans. ASME D, J. Basic Engng, 82.

Kupcis, E. A. (1990). Manually flown windshear recovery technique. In Proc. 29th IEEE Conf. on Decision and Control, Honolulu, HI, pp. 758-759.

Lane, S. H. and R. F. Stengel (1988). Flight control using non-linear inverse dynamics. Automatica, 24, 471-483.

Menon, P. K. A. et al. (1987). Nonlinear flight test controllers for aircraft. $J$. Guidance, 10, No. 1.

Menon, P. K. A. et al. (1991). A two-time-scale autopilot for high performnce aircraft. Presented at the 1991 AIAA Guidance, Navigation, and Control Conf., New Orleans.

Miele, A. et al. (1986a). Guidance strategies for nearoptimum takeoff performance in wind shear. J. Optim. Theory Applic., 50, No. 1.

Miele, A. et al. (1986b). Optimization and gamma/theta guidance of flight trajectories in a windshear. Presented at the 15th ICAS Congress, London.

Miele, A. et al. (1990). Optimal trajectories and guidance trajectories for aircraft flight through windshears. In Proc. 29th IEEE Conf. on Decision and Control, Honolulu, HI, pp. 737-746.

Mulgund, S. S. and R. F. Stengel (1993a). Target pitch angle for the microburst escape maneuver. $J$. Aircraft, 30, $826-825$.

Mulgund, S. S. and R. F. Stengel (1993b). Optimal recovery from microburst wind shear. J. Guidance, Control, Dyn., 16, 1010-1017.

Mulgund, S. S. and R. F. Stengel (1993c). Aircraft flight control in wind shear using partial dynamic inversion. In Proc. 1993 American Control Conf., San Francisco; also to appear in $J$. Guidance, Control, Dyn.

Oseguera, R. M. and R. L. Bowles (1988). A simple, analytic 3-dimensional downburst model based on boundary layer stagnation flow. NASA TM 100632, Washington, DC. 
Oseguera, R., R. Bowles and P. Robinson (1992). Airborne in situ computation of the wind shear hazard index. AIAA Paper 92-0291, Presented at the 30th Aerospace Sciences Meeting, Reno, NV.

Psiaki, M. L. (1987). Control of flight through microburst wind shear using deterministic trajectory optimization. $\mathrm{PhD}$ thesis, Department of Mechanical and Aerospace Engineering, Princeton University, Report No. 1787-T.

Psiaki, M. L. and K. Park (1992). Thrust laws for microburst wind shear penetration. J. Guidance, Control, Dyn., 15, No. 4.

Psiaki, M. L. and R. F. Stengel (1985). Analysis of aircraft control strategies for microburst encounter. J. Guidance, Control, Dyn., 8, 553-559.

Psiaki, M. L. and R. F. Stengel (1991). Optimal aircraft performance during microburst encounter. $J$. Guidance, Control, Dyn., 14, 440-446.
Singh, S. N. and W. J. Rugh (1972). Decoupling in a class of nonlinear systems by state feedback. ASME J. Dyn. Syst., Measurement, Control, Ser. G, 94, 323-329.

Stengel, R. F. (1990). Course Notes for MAE 566; Aircraft Dynamics, Princeton University.

Stengel, R. F. (1993). Toward intelligent flight control. IEEE Trans. Syst., Man, Cyber., CMC-23, 1699-1717.

Townsend, J. (1983). Low-Altitude Wind Shear and Its Hazard to Aviation. National Academy Press, Washington, D.C.

U.S. Department of Transportation (1987). Windshear Training Aid. Federal Aviation Administration, Washington, DC.

Zhao, Y and A. E. Bryson (1990a). Optimal paths through downbursts. J. Guidance, Control, Dyn., 13, 813-818.

Zhao, Y. and A. E. Bryson (1990b). Control of an aircraft in downbursts. J. Guidance, Control, Dyn., 13, 819-823. 